\title{
Automated Measurement of Sintering Degree in Optical Microscopy Through Image Analysis of Particle Joins
}

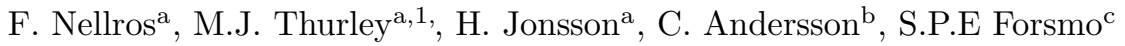 \\ ${ }^{a}$ Luleå University of Technology, Dep. of Computer Science, Electrical E Space Engineering, Luleå SE-97341, SWEDEN \\ ${ }^{b} R E D$, Luossavaara-Kiirunavaara AB,SE-98381 Malmberget, SWEDEN \\ ${ }^{c} M K 3$, Luossavaara-Kiirunavaara AB,SE-98381 Malmberget, SWEDEN
}

\begin{abstract}
In general terms, sintering describes the bonding of particles into a more coherent structure, where joins form between packed particles, usually as a result of heating. Characterization of sintering is an important topic in the fields of metallurgy, steel, iron ore pellets, ceramics, and snow for understanding material properties and material strength. Characterization using image analysis has been applied in a number of these fields but is either semi-automatic, requiring human interaction in the analysis, or based on statistical sampling and stereology to characterize the sample. This paper presents a novel fully automatic image analysis algorithm to analyze and determine the degree of sintering based on analysis of the particle joins and structure. Quantitative image analysis of the sintering degree is demonstrated for samples of iron ore pellets but could be readily applied to other packed particle materials. Microscope images of polished cross-sections of iron ore pellets have been imaged in their entirety and automated analysis of hundreds of images has been performed. Joins between particles have been identified based on morphological image processing and features have been calculated based on the geometric properties and curvature of these joins. The features have been analyzed and determined to hold discriminative power based on a statistical evaluation using the Welch t-test and display properties consistent with sintering theory and results from traditional pellet diameter measurements on the heated samples.
\end{abstract}

Keywords: image analysis, feature extraction, morphological image processing, curvature, sintering, microscopy, iron ore pellets

\section{Introduction}

Characterization of sintering is an important topic in numerous fields, including; metallurgy, ceramics, steel, iron ore, and snow. Sintering affects material properties, particularly strength, and describes the bonding of particles into a coherent structure typically via thermal treatment.

In the mining of iron ore, refining and concentration is performed to produce high-grade iron ore products that provide beneficial production conditions in steel making. One such product is iron ore pellets, where the refined and ground ore is balled into spheroids of about about $1 \mathrm{~cm}^{3}$ and then heat treated. The reason for this process is that the very strong pellets are more efficient to use than the finely ground powder they are made of, since they can be stacked in a pile and do not crumble at very high temperatures in the blast furnace, thus enabling a continuously high air flow through the entire reduction to pure iron [1].

The final strength of the pellet depends highly on the sintering of the iron-oxide hematite particles that take place during the heat treatment. In the case where the ore consist of magnetite iron-oxide, the nature of the sintering is dependent on the initial process of oxidizing the magnetite to hematite. Getting these processes to run in a balanced way is the key to creating a high quality end product [2]. To evaluate macro behavior during transportation and blast furnace reduction properties, pelletizing companies usually have large lab facilities that can work in micro and pilot scale. In these labs, standardized tests are performed to measure such things as cold compression strength and reducibility [1].

In the pelletizing labs, sintering degree is never measured but related properties, such as porosity and expansion during heat treatment and shrinkage during cooling $[1,3,4]$, can be measured. However, to really understand what

Email address: mjt@ltu.se (M.J. Thurley)

${ }^{1}$ Phone: +46730293132 
happens and why, one must study the micro structure. Up to now this has very much been a handcraft work, an art-form of the trained expert eye such as the PhD thesis by Niiniskorpi [2]. Microscope operators and researchers that have been working at the same pellet plant for decades can look in the oculars and evaluate the quality of the product that they see, having years of experience to judge from. However, these experts are increasingly rare with people changing jobs more frequently, and the need for quantitative, comparable measurements have risen. Furthermore, evaluating large amounts of pellets in a microscope is tiring for the human operator and the demand for reliable statistics and large amounts of data has increased.

In ceramics the topic of image analysis as a way of measuring sintering has been thoroughly explored. Chermant et al. [5] have been working with characterization of sintering through morphological analysis since the beginning of the 1980's [5, 6, 7]. Starting with metallurgical powders of convex particles in 1981, Chermant et al. have developed image analysis methods based on the stereology principles as developed by strong names such as DeHoff et al. [8], Serra [9]. The motivation of stereology is to assume randomness and by letting a grid, plane or line interact with a sample in $1 \mathrm{D}$ or $2 \mathrm{D}$ being able to unfold its corresponding $3 \mathrm{D} /$ volume properties through mathematical relationships. One typical example of a stereometric approach is the classical method within materials science called point counting.

The sintering of a metal powder is studied by Chermant et al. [5] in a time lapse sequence. Chermant et al. [5] performed a horizontal line scanning analysis on thresholded images to compute the connectivity, concavity and convexity numbers of particles. These three features were derived from the count of tangents in convex and concave parts of extreme points on the particle perimeters. Furthermore, necks that form between the particles were identified by an algorithm using the distance transform but this approach was only possible because particles were convex. The results were compared only to the conventional method of studying porosity and the conclusion was that sintering is possible to characterize in the stereometric measurements. Early publications following Chermant et al. [5] focus on validating that the stereometric analysis is indeed possible through automatic image analysis. Further methodology development investigate the full potential of the stereometric unfolding into 3D features such as integral mean curvature [6], i.e. the property corresponding to total curvature of pore-solid interface per unit volume, computed from a rescaled connectivity number.

More recent publications go into more advanced theory, such as relating the results to grain growth theory, performing simulations and investigation of sample homogeneity [7]. Focus lies on the sintering analysis and not full automation, and "images still need additional treatment" [7] which can be suspected to mean manual intervention in the preceding steps.

In steel and iron ore sinter cakes, image analysis has been used to study the porosity fraction and shape factors as an indicator of sintering degree $[10,11]$. Descriptions of the methodology are quite minimalistic and seem to contain some manual steps; e.g. "The void fraction and perimeter of voids was measured using commercial software for each binarised image." [10]. Kasai et al. [10] reported that the void fraction and specific surface area are correlated with strength of the sinter cake, and Molinari et al. [11] used image analysis methods to determine correct sintering time for steels with different boron contents. Later publications exist but seem mostly to vary the steel properties and not the image analysis methods [12].

The possible use of measuring curvature for quantifying sintering is also suggested for $3 \mathrm{D}$ data on snow. By consecutive slicing and imaging, Brzoska et al. [13] created 3D data of a wet snow sample in 1999. After segmenting the data into snow and pores, a method to measure local curvature from snow surface normal vectors and porosity medial axis was developed [13]. This technique has been further developed by Flin et al. [14] and explored using $\mu \mathrm{XRT}$ data to study curvature evolution over time and to model grain growth and the sintering process [15].

Only one previous publication on studies of sintering in iron ore pellets through image analysis have come to the author's knowledge. de Oliveira Simões et al. [16] claim to automatically identify and measure the necks and their number of connections and compare this to the pellets compressive strength. However, no method description on how this is performed in the Zeiss AxioVision software is given, and a visual inspection of the example segmentation shows clear ambiguities. Only three images at selected positions in each sample are analyzed. Results thus contain several unanswered questions and lack statistical validation but show an optimistic trend that there seems to be significant information in the analyzed measurements.

This paper presents an automated method for measuring sintering that can distinguish iron ore pellets subjected to different heat treatments through image analysis of cross-sections imaged in an optical microscope. One typical application of such a system would be lab scale evaluation of the sintering ability of new ores. The presented method studies necks and curvatures, however, not from the same stereological approach of Chermant et al. [5] but by directly identifying particle joins and analyzing their geometric properties. Data collection is semi-automated through Zeiss AxioVisions MosaiX module [17] and the image analysis is fully automated in MatLAB [18].

The results of this paper may interest several categories of readers such as both an image analysis audience and 
material scientists. Therefore the introduction is followed by a theory section that provides a short introduction to and review of both computational and material principles that have been applied. The method section has been supplemented with Appendix A, which contains a descriptive pseudo-code summary of the presented algorithm.

\section{Theory}

\subsection{Sintering}

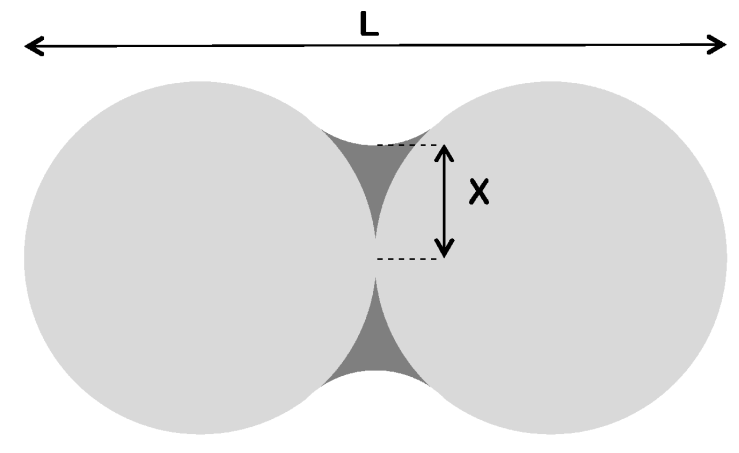

(a) Neck formation without particle merging. $x$ is the neck radius.

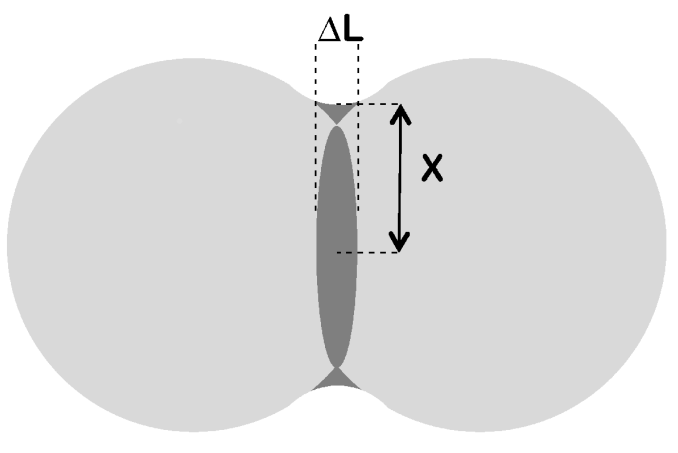

(b) Neck formation during particle merging. Shrinkage with a factor $2 \times \Delta L$.

Figure 1: Figure commonly used to describe the principle of sintering in two adjacent circular particles [19, 20].

Sintering is the bonding of particles into a more coherent structure (usually occurring under thermal treatment), where bridges start to form between particles at close range [19, 20]. Figure 1a depicts a simplified two particle model illustrating the initial stage for two adjacent spheres. Continuing the process, necks start to merge and grains fuse, see Figure 1b, which lead to a denser material interlaced with fewer but larger pores. Angularities become more round as particles diffuse and swell. Finally, pores entirely contained within particles, referred to as "closed porosity", disappear.

The optimum level of sintering for pellets is when necks have formed and increased the structural integrity, but when porosity is still uniformly high and allows a good gas flow throughout all of the pellet. Wynnyckyj and Fahidy [3] concludes that there is a drastic increase of crushing strength when shrinkage starts to be observed in the heated pellet, different from the steady and continuous growth of strength that can be measured initially. This most likely coincides with when neck growth turns into particle merging, as illustrated in Figure 1b.

\subsection{Image analysis}

The images are analyzed mainly through mathematical morphology techniques. These techniques are particularly useful for identifying and analyzing spatial structures in image data. Morphological image processing operations apply a structuring element, which is a spatial probe similar to a neighborhood operation, to the image. A short presentation of the operations used in this work is provided below, but or a more elaborate introduction to this topic, the reader is referred to the text Hands-On Morphological Image Processing by Dougherty and Lotufo [21] or any other standard text on image processing.

For a binary region $A$, erosion and dilation with a structuring element $B$ are easily defined with the set formulation given in Equations 1 and 2. Erosion results in a shrunken region in $A$, reduced to only the positions $x$ in which the translated structuring element $B$ fits. Dilation instead expands the region to include also the translation of $B$ to all positions $x$ within $A$.

$$
\begin{gathered}
i m e r o d e(A, B)=A \ominus B=\left\{x: B_{x} \subset A\right\}, \\
i m \text { dilate }(A, B)=A \oplus B=\bigcup\left\{B_{x}: x \in A\right\},
\end{gathered}
$$

where the subscripted $B_{x}$ denotes the translation of the origin of $B$ to the point $x$.

Combining dilation and erosion gives the two additional morphological operators of opening and closing, as defined by Equations 3 and 4 . An opening is the union of all translations of $B$ that fit inside $A$, resulting in a 
smoothed outer boundary of $A$, and enlarged intrusions and internal holes. Closing will instead smooth the outer boundary, shrink or fill internal holes smaller than $B$, and fill intrusions narrower than $B$.

$$
\begin{aligned}
& \operatorname{imopen}(A, B)=A \circ B=(A \ominus B) \oplus B \\
& \operatorname{imclose}(A, B)=A \bullet B=(A \oplus B) \ominus B
\end{aligned}
$$

Conditional dilations are also applied in this work where the result of $A \oplus B$ is restricted to only expand within another region, $C$, as mathematically described by Equation 5 .

$$
\text { imcondil }(A, B, C)=A \oplus_{C} B=(A \oplus B) \cap C
$$

If the conditional dilation is performed in several subsequent steps using the same structuring element, it is called a geodesic dilation. If the number of steps is infinite, i.e. the dilations and intersections are performed until they produce no more change in the result, the procedure is called a reconstruction of image $\mathrm{C}$ from $\mathrm{A}$ (Equation 6). $\mathrm{C}$ is referred to as the conditioning mask, and $\mathrm{A}$ is morphologically reconstructed into $\mathrm{C}$. The conditioning mask $\mathrm{C}$ is denoted in Equation 6 as a subscript below the dilation operation.

$$
\text { imreconstruct }(C, A)=C \nabla_{B} A=\left(A \oplus_{C} B\right)^{\infty}
$$

The term opening-by-reconstruction is used to denote an opening of an image $C$ by a given structuring element $D$, followed by the dilation reconstruction. In this case Image $A$ in Equation 6 would be given by the opening of $C$ by $D$.

The term closing-by-reconstuction is used to denote a closing of the image $C$ by a given structuring element $D$, followed by an equivalent reconstruction based on erosion equivalent to that described above in Equation 6 .

For grayscale images, these definitions are easily extended and the reader is referred to any standard text on image processing.

\subsection{Curvature}

Computing curvatures along a discrete outline in an image, essentially an arbitrary 8-connected path of pixels, is much different from finding curvatures along a derivable function as it entails also approximating derivatives [22, ch. 10]. The computation will unavoidably involve discrete points, and a precise way to choose these is therefore needed. In this work, the method for computing the curvature in a point of interest makes use of three points. Apart from the point itself, the end points of the two longest digitally straight segments (DSS:s) that extends backwards and forwards from the point of interest and along the outline, respectively, are used to compute the curvature. Such segments can be computed in linear time as shown by Debled-Rennesson and Reveillès [23].

The curvature is now the reciprocal of the radius $R$ of the circle defined by the points. This means that the curvature will be high when the outline around the point of interest has a sharp and angular shape while smooth and round outlines give raise to low curvatures, with the extreme case being a straight outline with zero curvature. As illustrated in Figure 2, the center of the circle is the intersection of the bisectors of the DSS:s, and $R$ is the Euclidean distance between the center and the point of interest. This way of computing the curvature was suggested by Hermann and Klette [24].

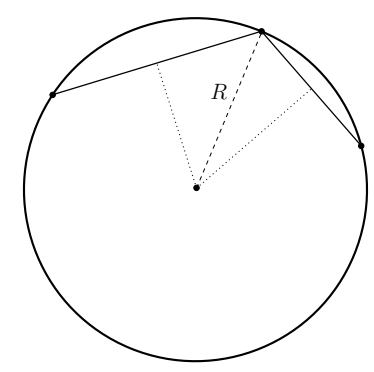

Figure 2: Principle for computing the curvature as $\frac{1}{R}$, where $R$ is the radius of a circle defined by the secants through three chosen points.

A equivalently curving concavity and convexity will have the same absolute curvature value. By keeping track of in which direction the backward and forward digitally straight segments are computed, and on which side of 
the path the circle center point lies on, a direction can be introduced and concavities can be assigned a negative value [24].

In their review of curvature based corner detectors, Kerautret et al. [25] emphasize blurred line segments [26] as common to a number of curvature estimators. Where the "blurred segment is a piece of thick digital straight line, whose width is the vertical diameter of its convex hull". Increasing the allowable width allows for a longer line that would be less sensitive to small noise variations in the digital line segment. In this work we evaluate the DSS curvature estimator and leave the blurred line segments for future work.

\section{Method}

The method is divided into the three sections: sample preparation, pellet imaging and image analysis. These are stand-alone processes performed separately in the order they are presented and are therefore best explained individually.

\subsection{Sample preparation}

A fine magnetite $\left(\mathrm{Fe}_{3} \mathrm{O}_{4}\right)$ iron ore concentrate was agglomerated into pellets via small scale laboratory balling as described by Forsmo et. al. [27]. As a binder, $0.5 \%$ Bentonite clay was used. The pellets were subsequently heat treated, i.e. oxidized to hematite $\left(\mathrm{Fe}_{2} \mathrm{O}_{3}\right)$, and sintered. The heat treatment was performed in a Setaram TMA96 and simultaneous dilation measurements were conducted. Dilation was determined with the use of Setarams Calisto software.

All pellets were heated in oxidizing atmosphere $\left(16 \% \mathrm{O}_{2}\right.$ in $\left.\mathrm{N}_{2}\right)$ with $5{ }^{\circ} \mathrm{C} / \mathrm{min}$ to different temperatures and held for different times to obtain a varied degree of sintering. The final temperatures were $1200{ }^{\circ} \mathrm{C}, 1250{ }^{\circ} \mathrm{C}$ and $1350{ }^{\circ} \mathrm{C}$. The sample heated to $1250^{\circ} \mathrm{C}$ was held for 30 minutes and two samples were heated to $1350{ }^{\circ} \mathrm{C}$, one was held for 0 minutes and one for 30 minutes.

The sample pellets were cast into epoxy, vacuum impregnated, cut in half and polished to give a good, reflecting cross section to study with the microscope. Microscopy images of each final sample are shown in Figure 3.

\subsection{Pellet imaging}

Images are retrieved with a Zeiss Axio Imager .M2m upright microscope system. The approach has been to use the routine MosaiX included in the AxioVison software [17] since it automatically retrieves images in the desired scanning pattern.

The user first creates a low magnification overview and on that marks the rectangular region from which the images are retrieved in a meander pattern. Images are retrieved after automatic focusing at each stage position and a defocused reference image is automatically subtracted to remove uneven illumination effects [28, vignetting correction] before saving. Initialization of the region is thus manual for each pellet, however the sequential image retrieval is unsupervised.

One pellet of $10 \mathrm{~mm}$ diameter results in around 700 12-bit grayscale images of size 1388x1040 pixels. Resolution lies at $0.32 \mu \mathrm{m}$ per pixel. Further processing through image analysis is fully automated and all necessary information is retrieved from the metadata .xml-file that is created by AxioVision.

\subsection{Image analysis}

Due to memory limitations and the desire to process the data at full resolution, images are processed individually. However, first the pellet region must be identified so that analysis only is performed at relevant regions and so that a local feature's distance to the pellet perimeter can be determined. The developed algorithm can be divided into 2 main parts:

1. identification of pellet region and global information

2. and processing of individual images;

providing the following outcomes where the sintering features are defined in step 8 on page 8 .

1. overview images of the pellet and its material phase distribution,

2. spatial distributions of sintering features and material phase data,

3. and statistical information about sintering features,

Based on the theory shortly introduced in Section 2.1 and previous work $[5,16]$, necks and curvature are chosen as the discriminative sintering properties. The exact outcomes are presented in Section 4. Analysis of the amount of oxidation in each pellet (conversion of magnetite to hematite) has been performed using repeated Otsu based histogram segmentation by Nellros and Thurley [29] with the results presented in Section 4.4. 


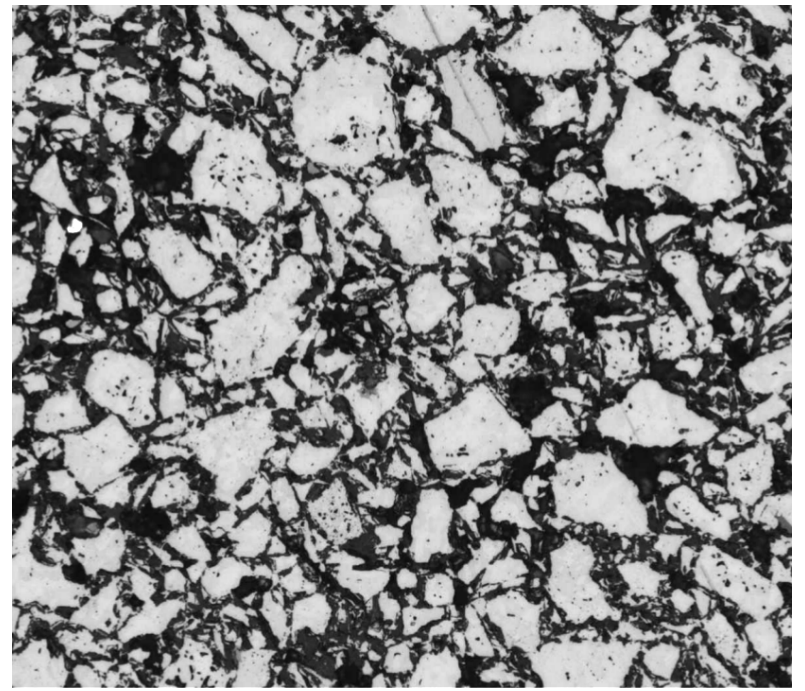

(a) A pellet heated to $1200^{\circ} \mathrm{C}$ and held for 0 minutes.

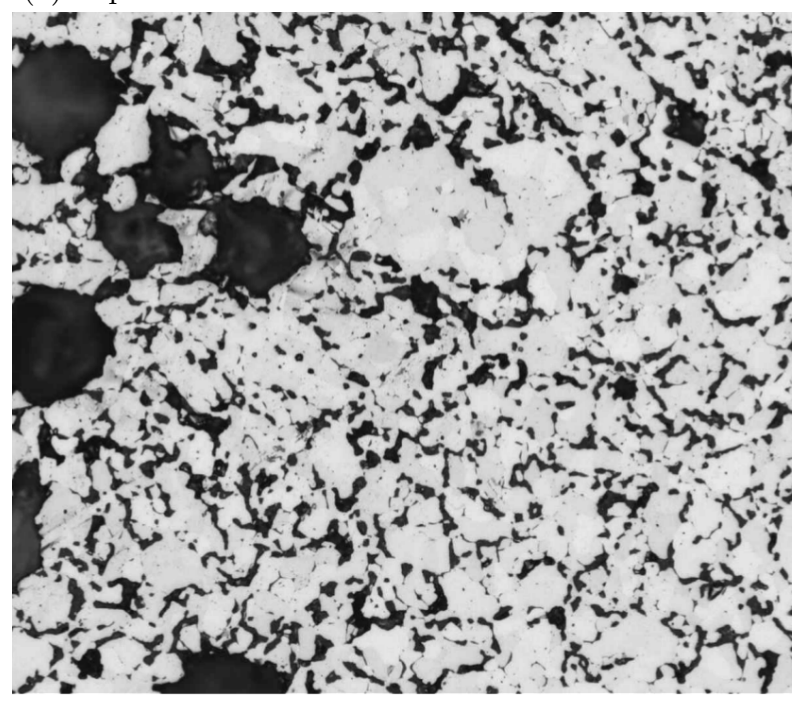

(c) A pellet heated to $1350{ }^{\circ} \mathrm{C}$ and held for 0 minutes.

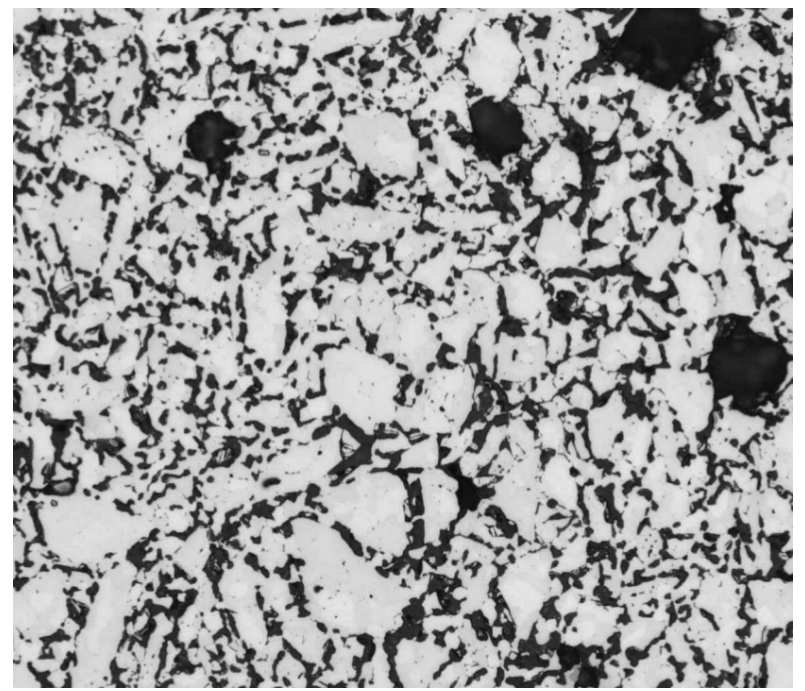

(b) A pellet heated to $1250{ }^{\circ} \mathrm{C}$ and held for 30 minutes.

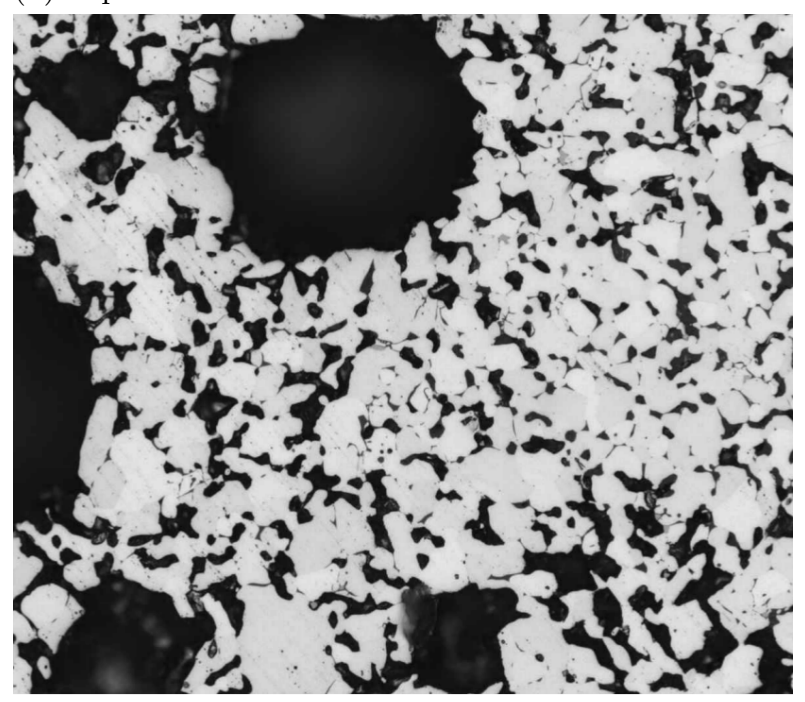

(d) A pellet heated to $1350{ }^{\circ} \mathrm{C}$ and held for 30 minutes.

Figure 3: Sample images of the micro-structures that can be observed in the four pellets after heat treatment. Note the differences in porosity (darker phase) size distributions, particle angularity and material density (brighter phase).

\subsubsection{Global analysis of the whole pellet}

The pellet region (Figure 4b) and segmentation thresholds for identifying magnetite and hematite are found by analyzing an overview mosaic such as Figure 4a using the morphological boundary detection algorithm presented by Nellros and Thurley [29].

\subsubsection{Processing of individual images}

To locate necks in each sub image, a number of sequential steps are performed. The position within the pellet for each sub image is traced by computing row and column number from the image number. The corresponding area from the binary mask (Figure $4 \mathrm{~b}$ ) is used to determine how much and what region of the image that belongs to the pellet of interest. Also, the distance transform [21, Ch. 3.11] of that binary pellet is used to determine the distance to the pellet perimeter for each pixel within the sub image. The image is finally binarised and necks can be identified and quantified.

The entire process is outlined in pseudo-code in Algorithms 1 and 2 in Appendix A and a more elaborate description is provided below, where numbering of steps is consistent with numbering of steps in the algorithms. An example image and some steps of the neck identification can be seen in Figure 5a. 


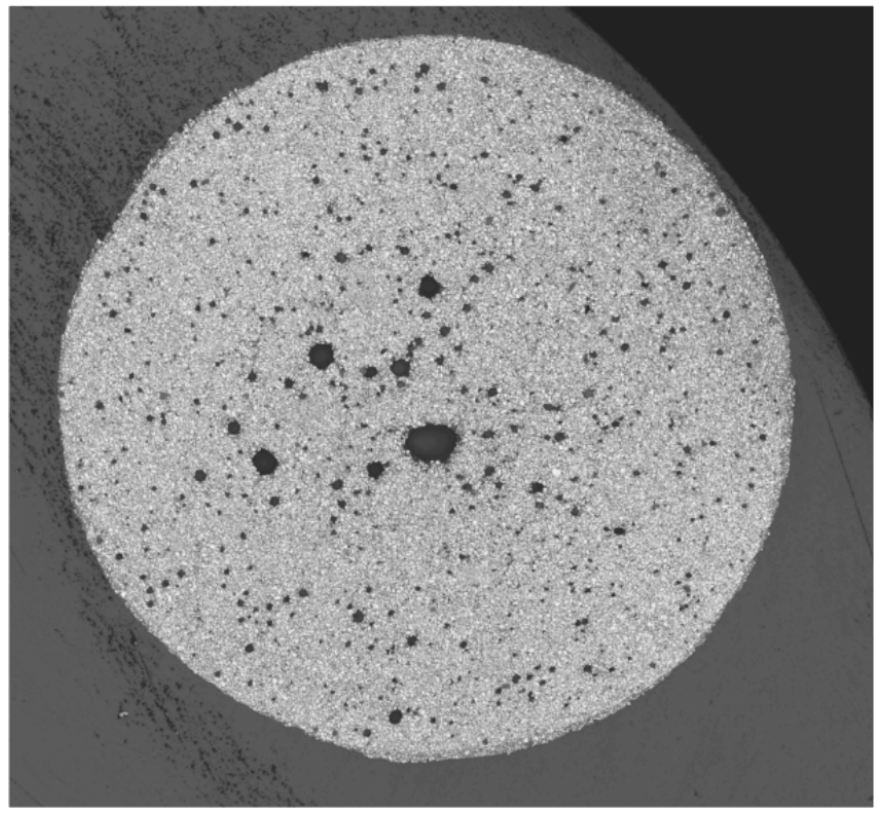

(a) An overview of the 1200 pellet (contrast enhanced).

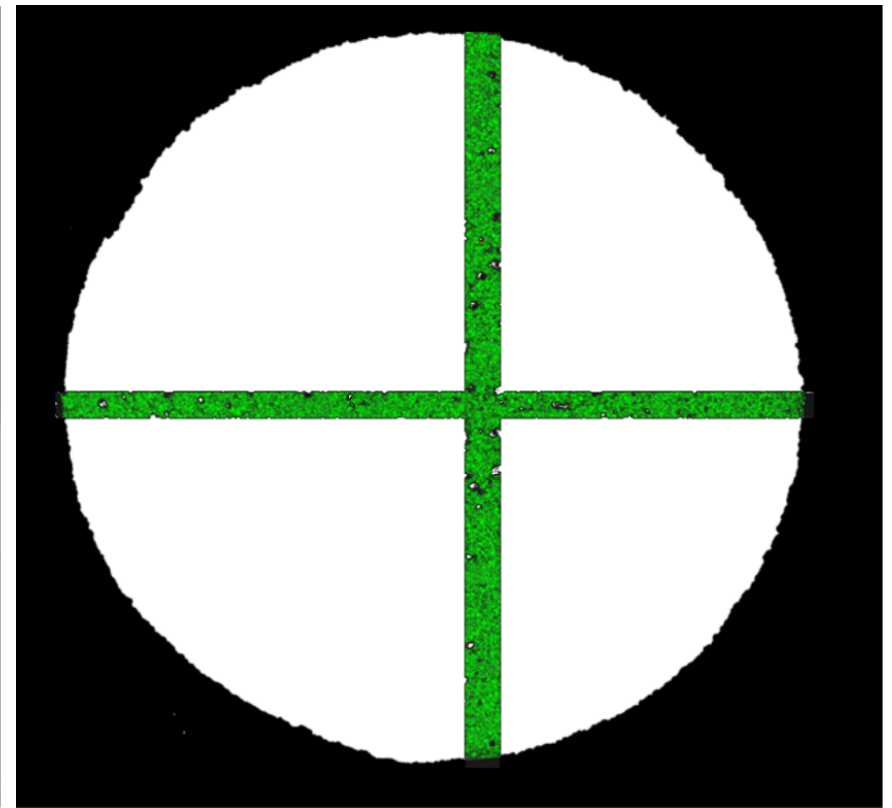

(b) The binary mask marking the pellet region for the 1200 pellet also showing the vertical and horizontal band of 48 images used to analysis this pellet.

Figure 4: An example pellet overview.

To reduce computational time, the neck analysis has been performed only on a vertical and horizontal stripe of 1-image-width approximately through the centre of each pellet (Figure 4b) comprising between between 46 and 51 images of 1388 by 1040 pixels for each pellet. The algorithm is not constrained by these limitations and presented in its general form. 46-51 images allows the identification of tens of thousands of particle joins (or "necks") and is considered sufficient to produce robust statistics.

1. Initialize. Compute image position from image number, assuming meander pattern and first image number to be 0 .

2. Load image. Load an image $I$ and retrieve corresponding images of pellet region mask and perimeter distances from global analysis.

3. Remove closed porosity and tiny particles. Smooth the image with a connected reconstructive alternating sequential filter as given by Equation 7 [21, Ch. 6.7, Eq 6.23].

This reconstructive-ASF filter applies a series of paired operations, closing-by-reconstruction then opening-byreconstuction using a common structuring element. The resulting image from each operation is used as both the input to the next operation and the conditioning mask for the morphological reconstuction. The subscript to the opening and closing operations in Equation-7 indicate the conditioning mask for the reconstruction. The structuring element is a diamond, $D$, with a radius $i$ that ranges from 1 pixel to $n$, where $n$ is the number of pixels corresponding to the smallest feature to be retained in the resulting image. In this work, the smallest feature (particle or hole) that we want to find is $3 \mu \mathrm{m}$ in diameter. Particles smaller than $3 \mu \mathrm{m}$ sinter very quickly and as a result are considered not significant to the testing.

$$
\begin{aligned}
A S F_{c o, D_{i}}^{n}\left(I_{0}=I\right) & =\left(\left(\left(\left(I_{0} \bullet_{I_{0}} D_{1}\right) \circ_{I_{c-1}} D_{1}\right) \ldots \bullet_{I_{o \_n-1}} D_{n}\right) \circ_{I_{I_{c-n}}} D_{n}\right), \text { where } \\
I_{c \_} n & =\left(I_{o \_} n-1 \bullet_{I_{O \_n-1}} D_{n}\right), \text { and } \\
I_{o \_} n & =\left(I_{c \_} n \circ_{I_{c-n}} D_{n}\right)
\end{aligned}
$$

This filter step will close internal porosity and remove small stray particles of size $3 \mu \mathrm{m}$ or smaller without affecting larger structures. Removing internal porosity is necessary to prevent the identification of false necks inside particles.

The effect of applying the reconstructive-ASF filter to an image can be seen for an example image in Figure 5b. Note that the filter is applied to a grayscale image and is smoothing out small grayscale variations which make 
the classification by grayscale value more robust. As the filter is reconstructive the particle boundaries in each image are reconstructed after each closing and opening operation. Therefore the selection of the diamond structuring element is not significant in terms of affecting the outer shape of particles and therefore will not impact the estimation of curvature.

4. Binarisation. Segment the filtered image into particle_phase (forground) and epoxy/porosity_phase (background) using Otsu's method [30].

5. Disconnect particles to identify potential necks. Perform consecutive openings [21, Ch. 2.1] on the binarised image with disks $C$ of radii $i$. The range of $i$ is set to the number of pixels corresponding to a chosen upper and lower limit of neck radii, here chosen as 1 and $5 \mu \mathrm{m}$.

$$
O_{i}=I \circ C_{i}
$$

The opening leaves the parts of the particles into which the structuring element fits, rounds off corners and cuts smaller bridges, which in this case corresponds to the necks.

6. Distinguish actual necks. For each opened image $O_{i}$, validate the properties of each region that has been removed by the opening. By defining a neck as a bridge that connects two (or more) regions that have not been removed by the opening, the necks can be distinguished from other regions removed by the opening, such as a bend or a sharp corner at the particle perimeter too small for the structuring element to have fit within.

7. Store neck raw data. If a region is validated to be a neck it is denoted $n_{j}$ and stored with the rest of the necks discovered by previous openings. A neck can be detected several times in the increasingly larger openings, as long as some of the two regions it bridges remain. In later quantification it is important to know which parts of the necks that were detected at which structuring element radius. Therefore, only the parts of $n_{j}$ that have not already been stored are added to necks. The incremental adding of regions for a neck detected by several openings is shown in Figure 6.

8. Quantify individual necks. By identifying each 8-connected component [21, Ch. 3.1] in necks (the combined regions discovered by the opening steps) as $n_{j}$, several measurements can be performed on the individual necks. Note that one component now can contain regions from several of the openings according to step 7 . The neck features chosen to quantify sintering are:

- area, simply as the number of pixels that make up $n_{j}$,

- neighbouring particle count, i.e. how many particles that the neck joins,

- detection radius (suggested as the equivalent measurement of the property $x$ in Figure $1 b$ ), the radius of the smallest structuring element that detected one of the regions that constitutes the neck component $n_{j}$,

- length - $L$ (or distance that the neck bridges, suggested as equivalent measure of property $\Delta L$ in Figure $1 \mathrm{~b}$ when shrinkage is occurring), defined as two times the average of the smallest distance from each connected particle (neighbor $n b$ in the set of all neighbors, $N B\left(n_{j}\right)$ ) to the neck centroid, $c_{j}$. The principle of computation for a three connected neck is given in Figure 7 and a mathematical formulation is provided in Equation 9.

$$
L\left(n_{j}\right)=\frac{2}{\left|\mathrm{NB}\left(n_{j}\right)\right|} \sum_{\mathrm{nb} \in \mathrm{NB}\left(n_{j}\right)} \min d\left(\mathrm{nb}, c_{j}\right)
$$

This seemingly complicated measure is chosen since necks often have more than two connections, and the length of a three way intersection (or more) is not as intuitively defined as in the ideal case of the two-connected neck in Figure 6.

- the distance to the pellet perimeter from the neck centroid is stored to be able to relate the above features to spatial position within the pellet.

9. Measure curvature. Finally, the curvature and the distance to the pellet perimeter is calculated for neck perimeter pixels neighboring pore phase, see Figure 6. Curvature is calculated with the method reviewed in Section 2.3 .

The choice of studying curvature only on neck perimeters is due to the hypothesis that the neck evolution is the measurement that quantifies sintering, and that along the necks, curvature should vary the most. 


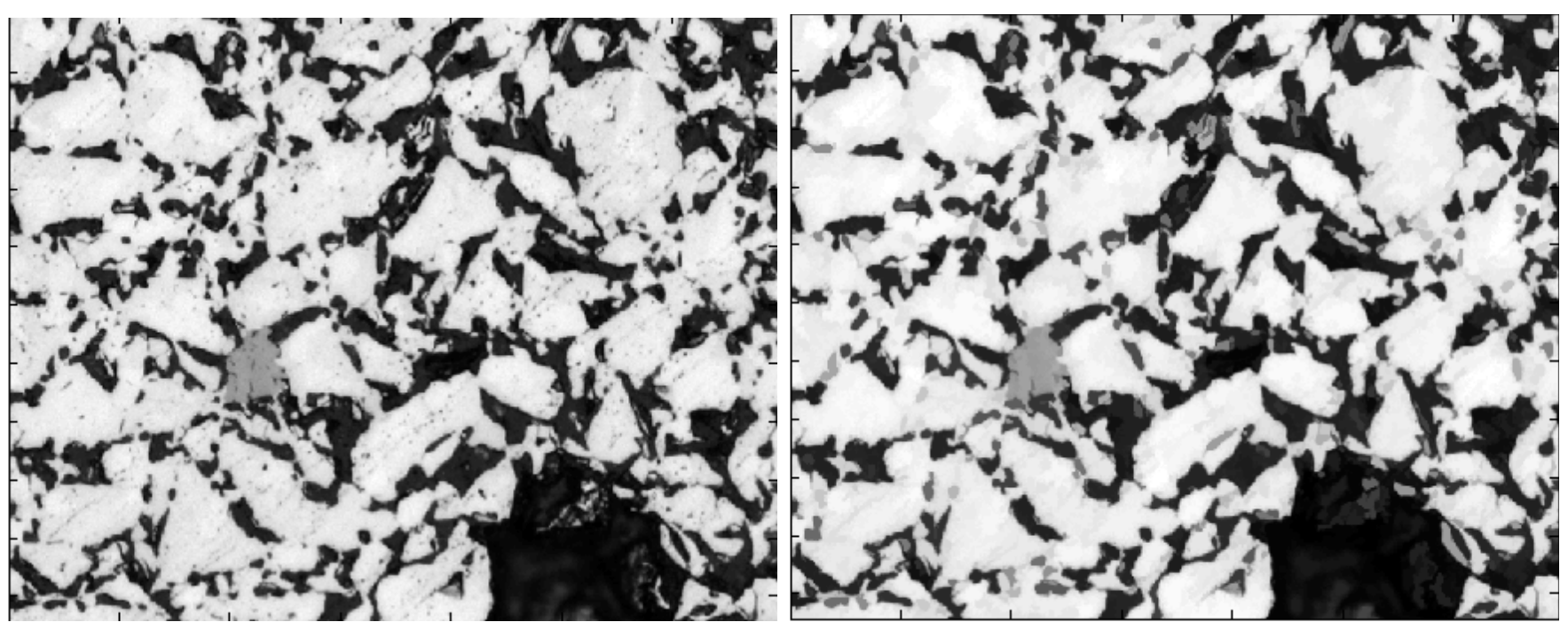

(a) Raw image straight from the microscope depicting hematite (b) The image in fig 5a after the connected alternating sequenparticles and, porosity and some residue rock (darker gray). tial filter. Closed porosity and tiny particles below $3 \mu \mathrm{m}$ have

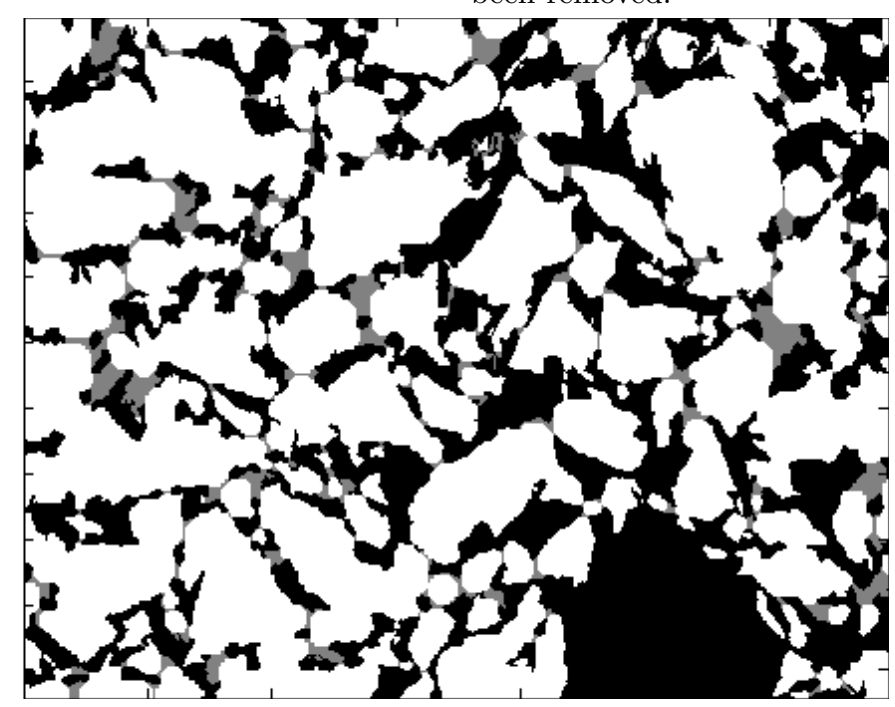

(c) The image in fig $5 \mathrm{~b}$ after thresholding, with identified necks overlaid in darker gray.

Figure 5: Selected steps in the identification of necks.

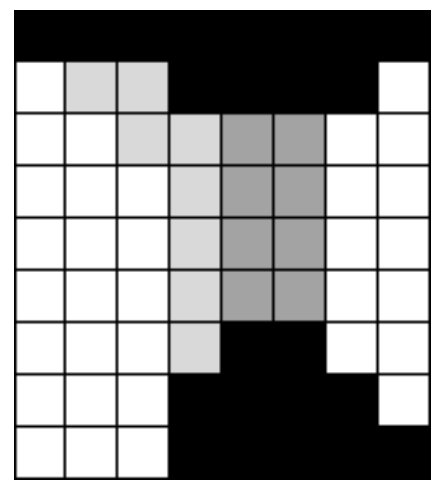

Figure 6: Image of a two-connected neck. The two gray shades correspond to regions identified at different opening radii in step 6 of the algorithm (page 8 ) and the white is the two surrounding particles. The black background is the pore phase. 


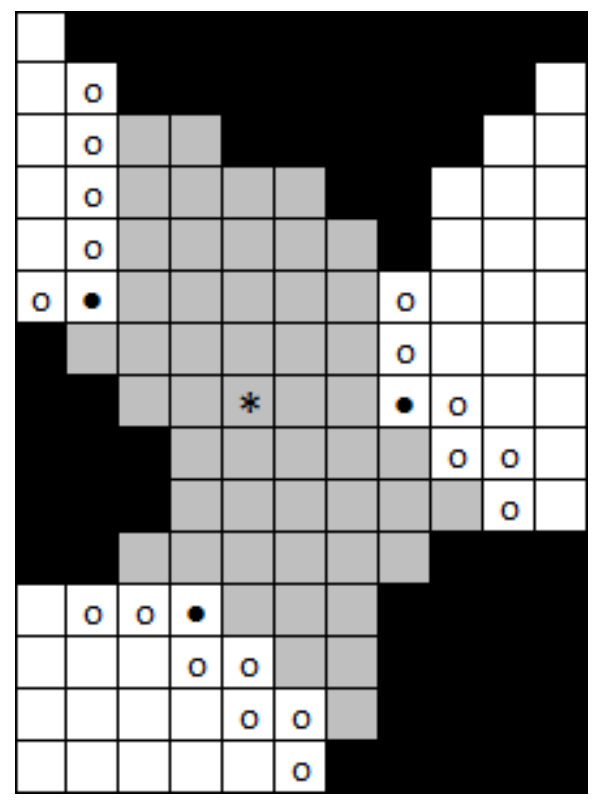

Figure 7: Image of a three-connected neck (shown in gray) between three particles (shown in white). Pixels involved in length calculation in algorithm step 8 (page 8 ) are marked. The centroid of the neck is marked with a $*$, particle perimeter pixels with $\mathrm{a} \circ$ and pixels determined closest to the centroid (used for the length computation) with a bullet.

\section{Results}

In figures and tables, the pellet samples are labeled with the maximum sintering temperature used and the holding time at this temperature in the following format temperature_holdingtime.

\subsection{Physical dilation measurements}

The accepted reference method for measuring shrinkage and expansion during heat treatment is a whole-ofpellet single diameter measurement of the pellet during heating which shall be referred to here as the "physical dilation" measurement. The physical dilation (diameter) of each pellet during heat treatment is shown in Figure 8. A reduction in the physical dilation measurement is expected as sintering occurs, and this is reflected in the graph by the decreasing diameter with increasing heat treatment.

\subsection{Statistical information}

The five features; detection radius, length, area, neighboring particle count and curvature, have been calculated for between 46 and 51 images of size 1388x1040 pixels for each pellet. Side-by-side box-plots for each feature visualizes variations for the four pellets. In addition, tables are provided, showing the number of necks (population size), the statistical measures; mean, median, standard deviation, variance and the 25 and 75 percent quartiles for each pellet, and the results of a statistical significance test.

The Welch t-test [31] is used to evaluate the statistical significance of the calculated features in there capacity to distinguish between the pellets. The Welch t-test is suitable for comparing populations that have unequal sample size, and unequal variance, to establish how different the populations are ( $\mathrm{t}$-value) and the probability ( $\mathrm{p}$-value) that this result could have been generated with random data. We assume each population of features follows a normal distribution.

The Welch t-test uses the following two Equations to calculate the t-value (Equation 10) and the degree-offreedom (d.f.) (Equation 11) for population $i$, where $\bar{X}_{i}$ is the mean, $s_{i}$ is the standard devition, and $n_{i}$ is the sample size. The p-value is calculated for a two tailed hypothesis using standard statistical tools based on the $\mathrm{t}$-value and the degree-of-freedom.

$$
t=\frac{\bar{X}_{1}-\bar{X}_{2}}{\sqrt{s_{1}^{2} / n_{1}+s_{2}^{2} / n_{2}}}
$$




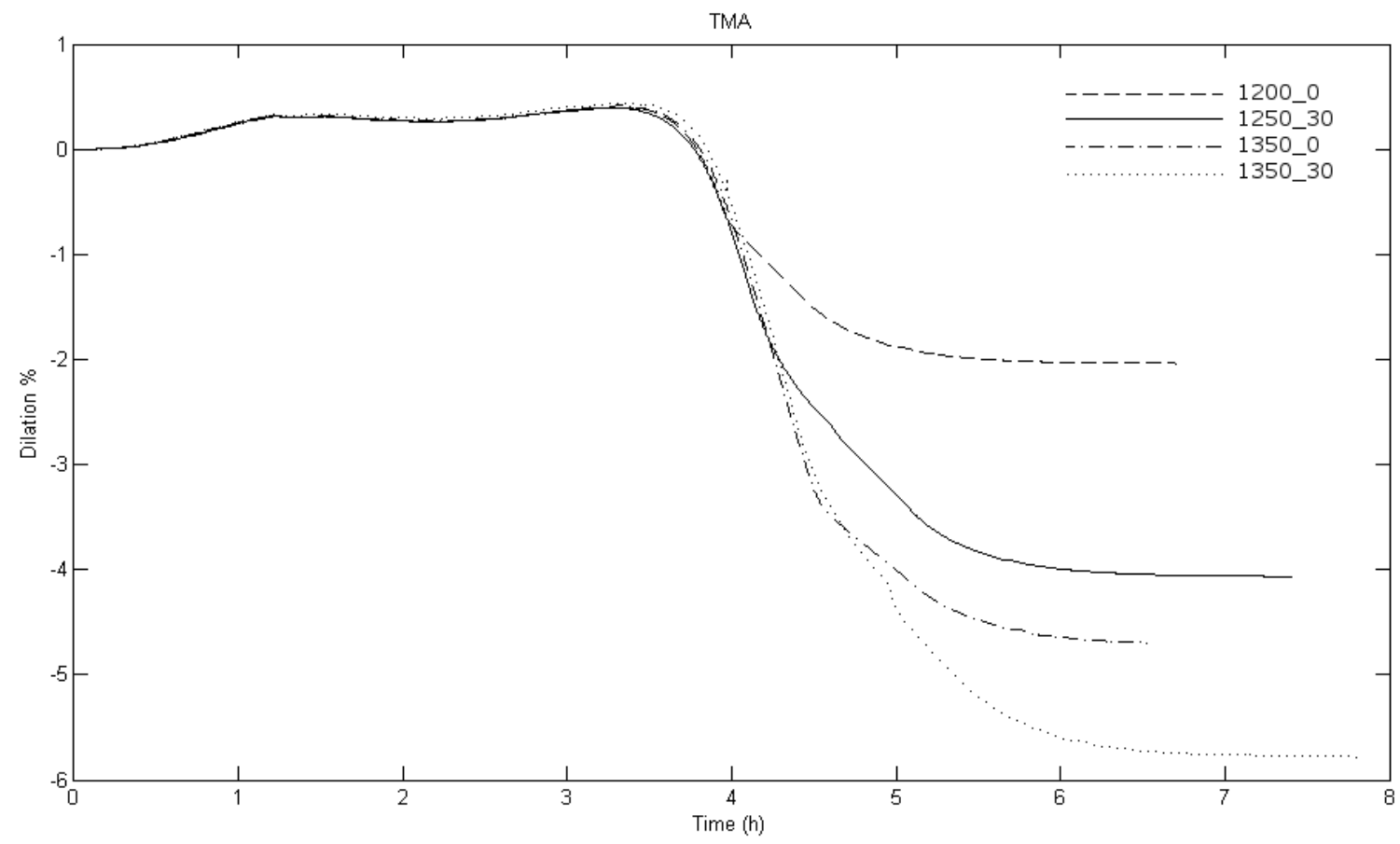

Figure 8: Physical dilation (diameter) curves for the four pellets during heat treatment in the Setaram TMA96, showing the degree of swelling and shrinkage in the pellet diameter over time.

$$
\text { d.f. }=\frac{\left(s_{1}^{2} / n_{1}+s_{2}^{2} / n_{2}\right)^{2}}{\left(s_{1}^{2} / n_{1}\right)^{2} /\left(n_{1}-1\right)+\left(s_{2}^{2} / n_{2}\right)^{2} /\left(n_{2}-1\right)}
$$

\subsubsection{Detection radius, length and area}

Figure 9 and Table 2 show a distinct increase in detection radii with increased heat treatment. The conclusions that can be drawn from this are discussed in Section 5.2.1.

Figure 10 and Table 3 show a more varied response in neck length statistics for the different pellets. Hypotheses concerning this are presented in Section 5.2.1. Note that the x-axis on Figure 10 is logarithmic.

Since a neck's area can be suspected to be highly correlated with its length and detection radius, these plots have been excluded. The trend that can be seen in the area is the same as for detection radius, increasing area with increasing heat treatment.

\subsubsection{Neighboring particle count}

The number of neighboring particles, is a discrete measure, ranging from 1 to 11 in this set of samples. The distribution of number of neighboring particles is shown for each sample in Table 6. From Table 5, the standard deviation of this feature is low and the vast majority of the necks only have two neighbors.

The most significant difference between the samples that can be observed in Table 6 are the higher valued outliers, meaning more complex connections, that slightly increase and then decrease again for the two most heat treated pellets.

\subsubsection{Curvature}

Studying Figure 11, curvatures seems to be almost normally distributed, except small shifts in the mean ranging from -0.2 to 0.1 . From Table 4 , it also seems that curvature slowly increases from negative to positive with increased temperature treatment, while standard deviation decreases. See Section 5.2.3 for a discussion of the indications of these results.

\subsection{Spatial information}

For the pellet in Figure 4, the spatial distributions of neck areas is shown in Figure 12. As can be seen, there is no visible variation within the pellet. The other features' spatial distributions look similar for all studied pellets 
and will therefore not be shown. Some discussion on conclusions about method robustness that can be drawn from the evenness of the spatial information are provided in Section 5.4.

\subsection{Material phase information}

The phase information has been calculated for the whole pellet (approx 700 images) to avoid bias from bubbles. In Figure 13 it can be seen that porosity fraction decreases with increased heat treatment. None of the pellets contain any residue magnetite and are thus fully oxidized.

\subsection{Neck density}

In addition to all the individual neck features, the average number of necks per $\mathrm{mm}^{2}$ has also been plotted in Figure 14. The trend that can be seen is an increase in number of necks from the least heated to the intermediate sample, and then a substantial decrease for the two most heated with the lowest values of all four.

\begin{tabular}{ccccccccccc}
\multicolumn{10}{c}{ Neck areas $\left(\mu \mathbf{m}^{2}\right)$} \\
\hline \hline$i$ & pellet & \# of necks & mean & std & q25 & median & q75 & \multicolumn{2}{c}{ Welch t-Test (row $i+1$ vs $i$ ) } \\
& & & & & & & & t value & d.f. & p value \\
\hline 1 & $1200 \_0$ & 24700 & 4.34 & 8.35 & 1.05 & 2.00 & 4.21 & 34.9 & 46790 & $<1 \mathrm{e}-05$ \\
2 & $1250 \_30$ & 30221 & 7.97 & 15.81 & 1.37 & 2.84 & 7.26 & 17.0 & 44082 & $<1 \mathrm{e}-05$ \\
3 & 1350_0 & 23652 & 10.67 & 20.06 & 1.68 & 3.79 & 10.21 & 10.8 & 34724 & $<1 \mathrm{e}-05$ \\
4 & 1350_30 & 17008 & 12.97 & 21.83 & 2.10 & 5.26 & 13.31 & - & - & - \\
\hline
\end{tabular}

Table 1: Statistical data for neck areas.

\begin{tabular}{|c|c|c|c|c|c|c|c|c|c|c|}
\hline \multicolumn{11}{|c|}{ Neck detection radii $(\mu \mathrm{m})$} \\
\hline$i$ & pellet & \# of necks & mean & std & $\overline{q q 25}$ & median & $\mathrm{q} 75$ & $\overline{\text { Welch } \mathrm{t}}$ & $\overline{\text { Test }(\mathrm{r}}$ & $\overline{i+1 \text { vs } i)}$ \\
\hline & & & & & & & & $\mathrm{t}$ value & d.f. & $\mathrm{p}$ value \\
\hline 1 & 1200_0 & 27400 & 1.31 & 0.66 & 0.97 & 0.97 & 1.30 & 64.4 & 54552 & $<1 \mathrm{e}-05$ \\
\hline 2 & 1250_30 & 30221 & 1.74 & 0.93 & 0.97 & 1.62 & 2.27 & 39.2 & 47573 & $<1 \mathrm{e}-05$ \\
\hline 3 & 1350_0 & 23652 & 2.08 & 1.05 & 1.30 & 1.95 & 2.59 & 36.9 & 33769 & $<1 \mathrm{e}-05$ \\
\hline 4 & 1350_30 & 17008 & 2.50 & 1.19 & 1.62 & 2.27 & 3.24 & - & - & - \\
\hline
\end{tabular}

Table 2: Statistical data for neck detection radii.

Neck lengths $(\mu \mathbf{m})$

\begin{tabular}{llllllllllcc}
\hline \hline$i$ & pellet & \# of necks & mean & std & q25 & median & q75 & \multicolumn{3}{c}{ Welch t-Test (row $i+1$ vs $i$ ) } \\
& & & & & & & & t value & d.f. & p value \\
\hline 1 & 1200_0 & 27400 & 2.05 & 1.49 & 1.03 & 1.62 & 2.55 & 10.2 & 55377 & $<1 \mathrm{e}-05$ \\
2 & 1250_30 & 30221 & 2.20 & 2.02 & 0.99 & 1.46 & 2.60 & 6.32 & 47199 & $<1 \mathrm{e}-05$ \\
3 & 1350_0 & 23652 & 2.32 & 2.31 & 0.99 & 1.41 & 2.62 & 3.35 & 35603 & 0.00270 \\
4 & 1350_30 & 17008 & 2.40 & 2.42 & 0.99 & 1.43 & 2.68 & - & - & - \\
\hline
\end{tabular}

Table 3: Statistical data for neck lengths.

\section{Discussion}

\subsection{Physical dilation measurements}

The results of the physical dilation measurements show more shrinkage with increase in temperature and holding time, consistent with the desired properties of increased sintering.

It is noticeable that there is a significant difference between the first two pellets, followed by a more similar interval between the dilation of the three last pellets. This confirms the visual impression that is given by Figure 3 , that there is very little sintering in the 1200_0 least heat treated pellet. This is expected and the reason for the low temperature and short holding time, chosen to create a pellet that is only just oxidized. 
Neck perimeter curvatures $\left(\mu \mathbf{m}^{-1}\right)$

\begin{tabular}{llccccccccc}
\hline \hline$i$ & pellet & \# of necks & mean & std & q25 & median & q75 & \multicolumn{3}{c}{ Welch t-Test (row $i+1$ vs $i$ ) } \\
& & & & & & & & t value & d.f. & p value \\
\hline 1 & $1200 \_0$ & 27400 & -0.16 & 0.71 & -0.54 & -0.15 & 0.21 & 42.6 & 52517 & $<1 \mathrm{e}-05$ \\
2 & $1250 \_30$ & 30221 & 0.07 & 0.57 & -0.23 & 0.02 & 0.34 & 4.25 & 52625 & $2.2 \mathrm{e}-05$ \\
3 & $1350 \_0$ & 23652 & 0.09 & 0.52 & -0.18 & 0.05 & 0.33 & 0 & 38240 & 1 \\
4 & $1350 \_30$ & 17008 & 0.09 & 0.48 & -0.15 & 0.04 & 0.28 & - & - & - \\
\hline
\end{tabular}

Table 4: Statistical data for neck perimeter curvatures.

\section{Number of neighboring particles}

\begin{tabular}{lcccccc}
\hline \hline pellet & \# of necks & mean & std & q25 & median & q75 \\
\hline 1200_0 & 27400 & 2.07 & 0.33 & 2.00 & 2.00 & 2.00 \\
$1250 \_30$ & 30221 & 2.13 & 0.47 & 2.00 & 2.00 & 2.00 \\
$1350 \_0$ & 23652 & 2.17 & 0.53 & 2.00 & 2.00 & 2.00 \\
$1350 \_30$ & 17008 & 2.17 & 0.49 & 2.00 & 2.00 & 2.00 \\
\hline
\end{tabular}

Table 5: Statistical data for neighboring particle count.

Distribution of the number of connecting neighbor particles for each neck

\begin{tabular}{llcccccccccccc} 
pellet & \# images & \# of necks & \multicolumn{4}{c}{ Number of necks connecting the given number of neighbor particles } \\
\hline \hline & & & 1 & 2 & 3 & 4 & 5 & 6 & 7 & 8 & 9 & 10 & 11 \\
\hline $1200 \_0$ & 48 & 27400 & 219 & 25254 & 1670 & 214 & 38 & 4 & 1 & 0 & 0 & 0 & 0 \\
$1250 \_30$ & 51 & 30221 & 184 & 26724 & 2638 & 493 & 131 & 34 & 12 & 1 & 1 & 2 & 1 \\
$1350 \_0$ & 46 & 23652 & 67 & 20493 & 2402 & 480 & 138 & 41 & 21 & 8 & 1 & 1 & 0 \\
$1350 \_30$ & 49 & 17008 & 49 & 14700 & 1777 & 365 & 80 & 32 & 5 & 0 & 0 & 0 & 0 \\
\hline
\end{tabular}

Table 6: Raw data for neighboring particle count - number of necks with a given number of connecting particles (neighbors) in each sample. 


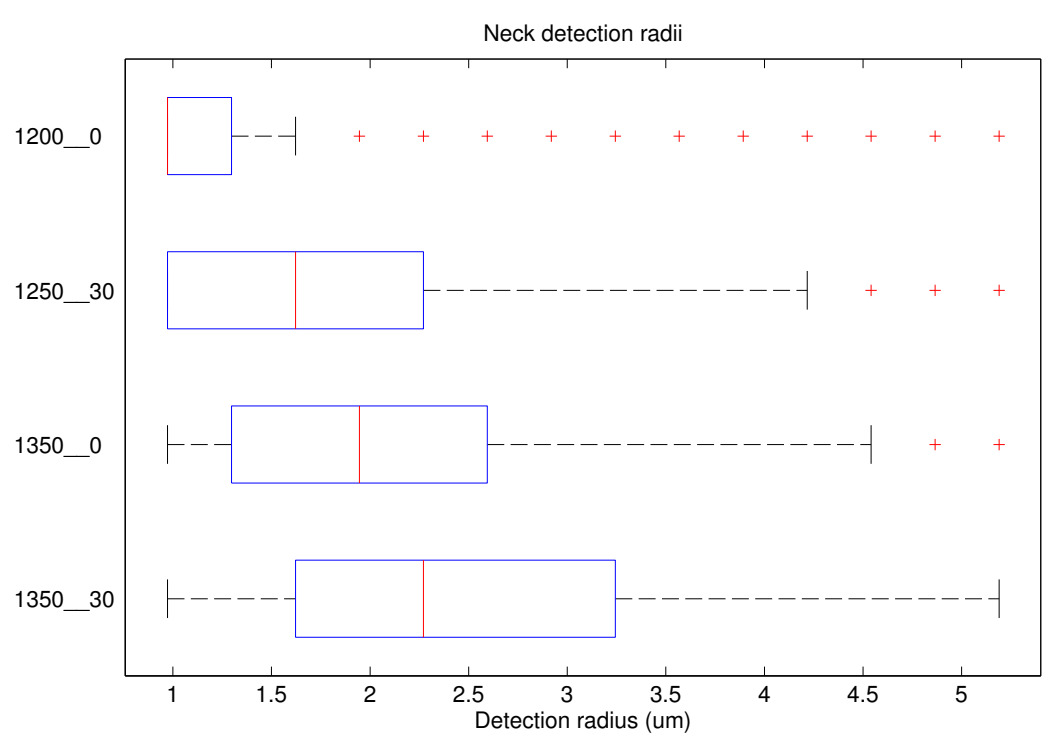

Figure 9: Box-plot of the detection radii of all necks in all four pellets.

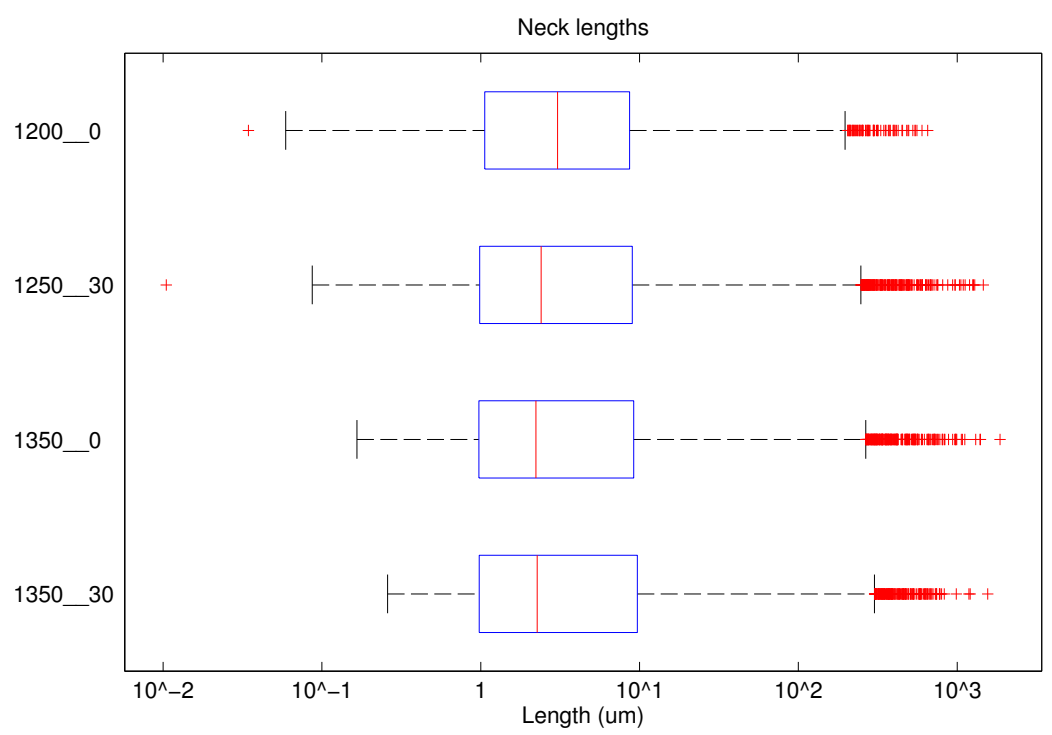

Figure 10: Box-plot of the length of all necks in all four pellets.

\subsection{Statistical information}

Based on the results of the Welch t-test and using a p-value threshold for significance of less than 0.01, we conclude that with one exception, all of the results are statistically significant. Therefore, the means of the populations are significantly different between the compared tests. The exception is the comparison of neck curvature between pellets 1350_0 and 1350_30 which were both heated to the same temperature and have equal mean. The primary reason for the high t-values and low p-values is the very large sample sizes.

Returning to Figure $1 \mathrm{~b}$ and the theory behind it, there are three processes that hypothetically can be assumed to affect the neck features. Initial neck formation, neck merging and, finally, particle merging and shrinkage.

\subsubsection{Area, length and detection radius}

The above three processes contribute to a monotonic increase in neck size with longer and higher temperature treatments, leading to more dense micro-structures. For the measured features area and detection radii, this would directly correspond to an increase with increased temperature, which can be confirmed in the result Tables 2 and 1 and in Figure 9. 


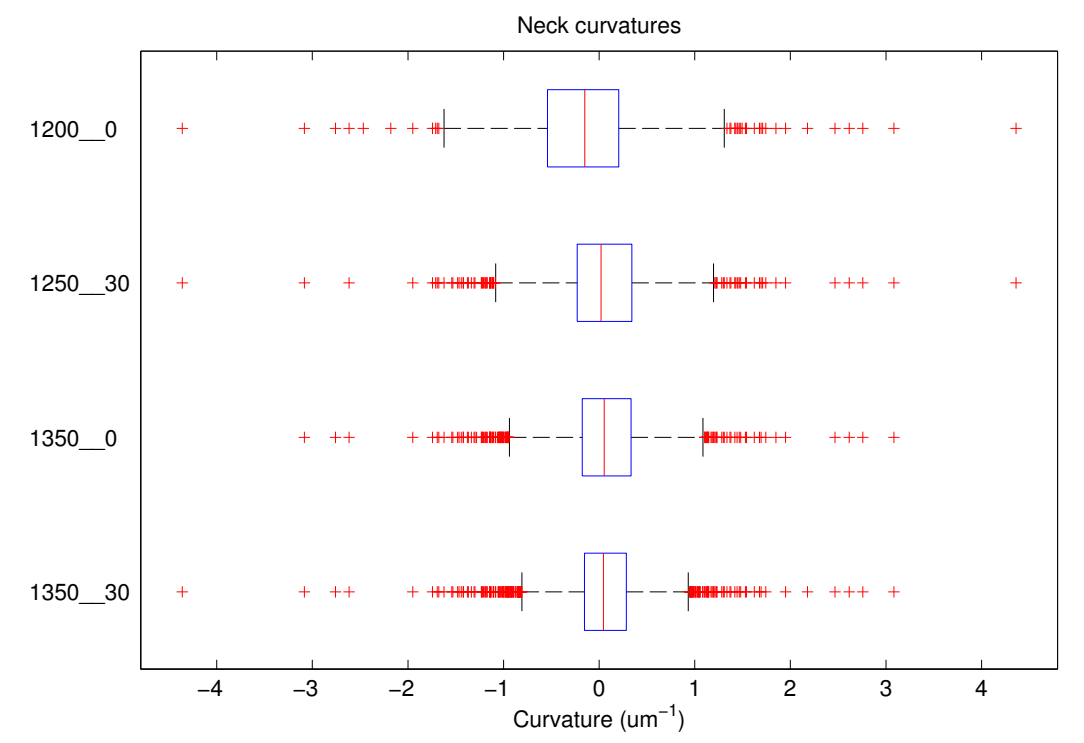

Figure 11: Box-plot of the curvature of all neck perimeters in all four pellets.

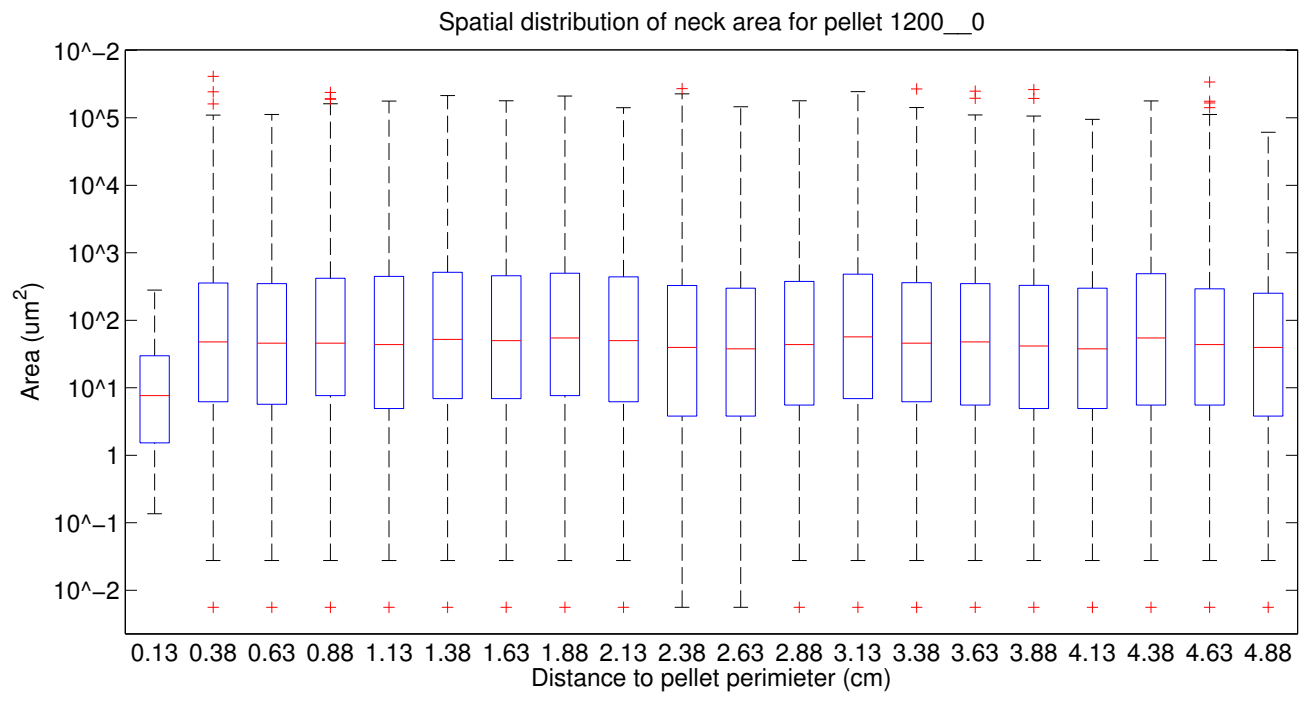

Figure 12: Spatial distributions of neck area for the two analyzed stripes for the pellet in Figure 4 . To ease interpretation, data has been grouped into boxes and the y-axis is in logarithmic scale.

The initial thought might be that the monotonic increase should also hold for the length, and that the results in Figure 10 and Table 3 are a testimony that something has failed in either assumption or computation, since the mean of the neck length increases slightly while the median slightly decreases with the increases in temperature and holding time. However, the authors believe that the effect of shrinkage and particle merging can explain the ambiguities in the length measurements.

As particles start to merge, the micro-structure becomes more dense and shrinkage can be observed even on macro scale. This means that the distance between particles decrease with increasing heating and holding time after shrinkage has started to occur. Also, particle structures become larger and rounder, causing the circular structuring elements to fit better and "dig deeper" into the necks during opening. These two processes counteracts the intuitive thought of necks bridging longer distances and is a reasonable explanation for the inconclusiveness with similar values and no distinct trend in the variations that can be seen in Figure 10 and Table 3.

It is clear that the chosen definition of length is a feature that does not quantify sintering, and for future work alternate definitions or another type of feature could be considered. 


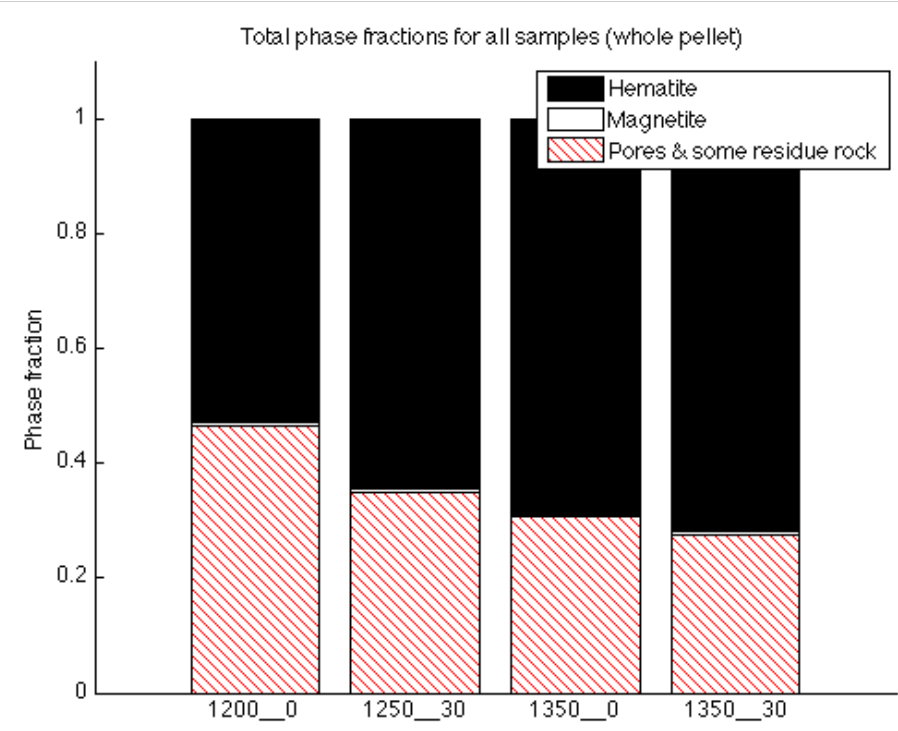

Figure 13: Fraction of the three phases hematite, magnetite and pores \& some residue rock in all four samples. Fractions of whole pellet area and not just the horizontal and vertical stripe.

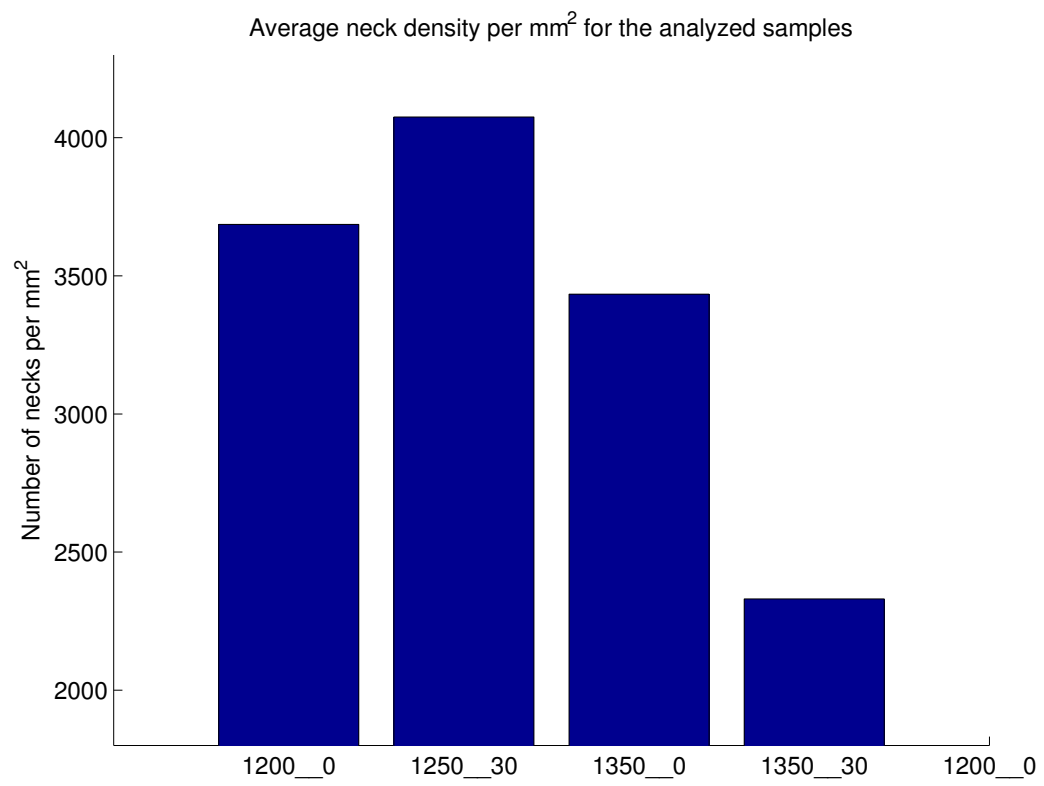

Figure 14: Number of necks per $\mathrm{mm}^{2}$ (Note the y-axis starts at 2000).

\subsubsection{Neighboring particle count}

Variation of number of neighboring particles is very low and most commonly equal to two, as presented in Tables 5 and 6 . It is not unreasonable that most necks indeed only bridge two different particles. When merging starts to occur, this should be between adjacent necks that bridge the same particle borders, meaning that necks still in most cases still connect only two particles.

Studying the outliers in Table 6 , there is a possible confirmation that neck and particle merging has started to occur in the two most sintered pellets, based on the trend with an increase in complexity of connections from the least sintered to the next pellet followed by a decrease in the two pellets with the most intense heat treatment, possibly related to the merging. 


\subsubsection{Curvature}

Curvature mean and distribution shifts from negative to slightly positive with increased heat treatment. Standard deviation decreases monotonically. Remembering that negative curvature means concavities, positive curvature means convexities and that the higher the absolute value of curvature, the more angular the particles, this matches the physical transformation that particles are assumed to be subject to according to sintering theory. Swelling causes fewer concavities, and the increased roundness lowers extreme values.

Starting from angular, irregular particles with few and sharp necks in the least heat treated pellet, observations should indeed be curvatures of both positive and negative values and a relatively large spread, which we see in Figure 11 and Table 4. As heating proceeds, particles merge, swell and become more convex. Concavities only exist in the necks, and the more the necks merge, the less negative curvatures become, since a straight line has curvature zero. As sintering continues, the micro-structure becomes more and more homogenous and curvature deviation should decrease, which also can be observed in Figure 11 and Table 4.

\subsection{Overview measures}

\subsubsection{Phase info}

The phase fractions per pellet presented in Figure 13 can be considered to correspond to a traditional measure that could indicate sintering degree. A lower porosity means more sintering, and there is a significant decrease with increased heat treatment in the studied pellet. All pellets are fully oxidized, which is beneficial for the sintering behavior.

It is noticeable that the porosity is slightly high compared to expected values around $30 \%$. There are three contributing factors that can explain this. Firstly, inter-particle porosity is added by the image analysis routine since phases are measured before applying the alternating sequential filter. Secondly, any non iron oxides may have been classified as porosity depending on their reflective properties in the microscope. Finally, the studied pellets were not ideally polished close to the pellet perimeters and the dark scratches increase the porosity count.

\subsubsection{Neck density}

Initial neck formation should contribute to an increase in neck density as long as no merging has started to occur. When shrinkage can be seen, existing necks below the chosen limit of $10 \mu \mathrm{m}$ gets fewer and fewer and the higher and longer heat treatment the pellets are subjected to, the lower the density should get.

For the studied pellets, the trend in Figure 14 with an initial increase follow by a very obvious decrease (almost halving the necks per $\mathrm{mm}^{2}$ ) supports the hypothesis that particle merging has started to occur in the two most heated pellets. Initial neck formation seems to occur for the two least treated pellet.

\subsection{Algorithm robustness}

Since the studied pellets are prepared in a process designed to create homogenous properties, the evenness of spatial feature distributions, as can be observed for neck area in Figure 12, is not a disappointing lack of information but a validation of the method. A future further validation could be to study less homogenous pellets and make sure that the algorithm detects the variations in structure.

The results of the phase segmentation presented in Figure 13 validates the increased sintering degree in the different pellets through the classical measurement of decreasing porosity.

The only arbitrary input in the image analysis algorithm is the resolution of the alternating sequential filter and the interval for possible detection radii of the necks. These have been chosen based on the properties of the raw material and the knowledge of experienced researchers.

Recent results [32] indicate that the theoretical limit of accuracy of determining curvature in digitized images could be as high as $40 \%$, and this should be kept in mind. Especially here where most neck widths are in the range of a few pixels only.

\subsection{Future work}

To determine how the results of this work can be fully utilized, future work should concern more measurements on pellets with know macro-structure properties and less homogenous structures. By doing this, results can be validated and continued analysis that can correlate the presented microscopy features with traditional pellet properties such as cold compression strength can be developed.

A faster implementation will allow processing of more of the image data. MATLAB is not the fastest solution, and as previously mentioned only between 46 and 51 images have been analyzed for the sintering related features for each pellet. The validation of robustness from the spatial information justifies that these results are representative, but if less homogenous pellets are analyzed in the future this might not be the best way of choosing a subset of 
images. However, the method supports any type of subset, such as a random, taking image numbers as an optional argument to constrict the analysis to a subset.

Using blurred line segments [26] to find the line segments to use in the curvature estimator will likely provide more discrimination in the curvature estimator and may provide some further insight.

\subsection{Concluding remarks}

The features neck area, detection radii and curvature as well as the neck density definitely hold discriminative power. Differences between the four pellets are large and trends in the features are coherent with theory on sintering, showing for increased heat treatment;

- increased neck detection radii and area,

- a more positive and less varied curvature, and

- initial increase followed by a decrease in neck density.

The results are not only consistent with assumptions based on theory but also with the actual shrinkage of the pellets during heating. The inter-mutual relationship between the shrinkage percentages are reflected in the means of neck area, detection radii and curvature, with a larger difference between the two least heat treated samples, and closer numbers when looking at the three most heat treated. The individual trends for each of these features can also be related to the monotonically increasing shrinkage.

In this work, two-dimensional cross-sections have been chosen for the study. All measurements, but perhaps neighboring particle count especially, are measurements of a much more complicated process in three dimensions. This complexity should not be underestimated when evaluating the results and has been in mind throughout in this work.

\section{Conclusion}

This paper has presented an automatic image analysis method to quantitatively determine sintering degree based on the structure and geometry of particle joins.

The method is a based on general knowledge of sintering and is therefore possible to apply on other packed particle materials after only small adaptations. There are only two arbitrary inputs to the algorithm - the resolution of the smallest included features and the interval for possible detection radii of the necks. These are chosen based on the properties of the raw material and can be easily obtained from knowledge of experienced researchers within the field.

The algorithm has been tested on microscope images of polished cross-sections of iron ore pellets. These have been analyzed using morphological image processing to identify the particle joins. For each join, the approach has been to measure some predefined features derived from sintering theory. By comparing the features for four pellets subjected to different heat treatments in a controlled environment, the four features area, detection radii, curvature and density have been determined to hold discriminative power by displaying properties consistent with sintering theory and results from traditional dilation measurements.

\section{Acknowledgments}

This work has been funded by the Hjalmar Lundbohm Research Centre (HLRC).

There are several people at LKAB that have been involved in sample preparation. Their efforts are appreciated and the authors would like to thank them one and all, but especially Gustaf Magnusson and Linn Sävlind. 


\section{Appendix A. Algorithms}

Algorithm: Binarisation

Constants:

$\mathrm{N}$ - image number,

$\mathrm{J}$ - number of image columns,

$\frac{\mathrm{k}}{\mathrm{pc}}-\mu \mathrm{m}$-limit $\mathrm{k}$ in number of pixels,

$\mathrm{D}_{\mathrm{i}}$ - diamond structuring element, radius $i$ pixels,

$C_{i}$ - circular structuring element, radius i pixels,

for each image I numbered $\mathrm{N}$

1. $\operatorname{row}=\left\lfloor\frac{\mathrm{N}}{\mathrm{J}}\right\rfloor+1$

For alternate rows scanning direction is opposite:

switch row $\bmod 2==0$

case 0

$\mathrm{col}=\mathrm{J}-\operatorname{rem}(\mathrm{N}, \mathrm{J})$

otherwise

$\mathrm{col}=\operatorname{rem}(\mathrm{N}, \mathrm{J})+1$

end

2. pellet_region $=\operatorname{pelletMask}($ row, $\mathrm{col})$

distance_values $=$ distancePellet $($ row, $c o l)$

3. for $D_{i}, i=1: \frac{3}{p c}, I_{0}=I$

$I_{c}=\operatorname{imclose}\left(I_{i-1}, D_{i}\right)$

$I_{c}=\operatorname{inv}\left(\operatorname{imreconstruct}\left(\operatorname{inv}\left(I_{i-1}\right), \operatorname{inv}\left(I_{c}\right)\right)\right)$

$I_{o}=\operatorname{imopen}\left(I_{c}, D_{i}\right)$

$I_{i}=\operatorname{imreconstruct}\left(I_{c}, I_{o}\right)$

end

4. $\quad I_{i} \stackrel{\text { thresholding }}{\stackrel{\text { Otsu }}{\longrightarrow}}$ particle_phase, porosity_phase

particle_phase is the binarised image of particles

$\cdots$ 
Algorithm: (CONt.) - NeCK Detection And quantification

$\cdots$

5. for $C_{i}, i=\frac{1}{p c}: \frac{5}{p c}$

$\mathrm{O}_{\mathrm{i}}=$ imopen(particle_phase, $\mathrm{C}_{\mathrm{i}}$ )

potential_necks $=$ particle_phase $-0_{i}$

6. for $\mathrm{n}_{\mathrm{j}} \in$ potential_necks

7. necks $=$ necks $\cup \mathrm{n}_{\mathrm{j}}$ end

end

end

Individual neck quantification:

8. for $\mathrm{n}_{\mathrm{j}} \in$ necks

area $=$ numpixels $\left(\mathrm{n}_{\mathrm{j}}\right)$

num_conn $=\left|\mathrm{NB}\left(\mathrm{n}_{\mathrm{j}}\right)\right|$

detection_radius $=\min _{i}\left(C_{i}\left(n_{j}\right)\right)$

perimeter_distance $=$ distance_values $\left(c_{j}\right)$

length $=\frac{2}{\left|\mathrm{NB}\left(\mathrm{n}_{\mathrm{j}}\right)\right|} \cdot \sum_{\mathrm{nb} \in \mathrm{NB}\left(\mathrm{n}_{\mathrm{j}}\right)} \min \mathrm{d}\left(\mathrm{nb}, \mathrm{c}_{\mathrm{j}}\right)$

end

Neck perimeter curvature:

9. neck_perimeter $=$ necks $\cap$ perim(particle_phase)

curvatures = compute_curvature(neck_perimeter)

curvature_distances $=$ distance_values(neck_perimeter)

end 


\section{References}

[1] K. Meyer, Pelletizing of iron ores, volume 169, Springer-Verlag, Berlin, Heidelberg, New York, 1980.

[2] V. Niiniskorpi, Development of phases and structures during pelletizing of Kiruna magnetite ore, Ph.D. thesis, Åbo Akademi University, 2004.

[3] J. Wynnyckyj, T. Fahidy, Solid state sintering in the induration of iron ore pellets, Metallurgical and Materials Transactions B 5 (1974) 991-1000.

[4] S. Forsmo, S. Forsmo, P. Samskog, B. Björkman, Mechanisms in oxidation and sintering of magnetite iron ore green pellets, Powder technology 183 (2008) 247-259.

[5] J.-L. Chermant, M. Coster, J.-P. Jernot, J.-L. Dupain, Morphological analysis of sintering, Journal of Microscopy 121 (1981) 89-98.

[6] J. Chermant, Characterization of the microstructure of ceramics by image analysis, Ceramics international 12 (1986) 67-80.

[7] M. Coster, X. Arnould, J. Chermant, L. Chermant, T. Chartier, The use of image analysis for sintering investigations: The example of $\mathrm{CeO}_{2}$ doped with $\mathrm{TiO}_{2}$, Journal of the European Ceramic Society 25 (2005) $3427-3435$.

[8] R. DeHoff, F. Rhines, U. of Florida, Quantitative microscopy, McGraw-Hill series in materials science and engineering, McGraw-Hill, New York, 1968.

[9] J. Serra, Image analysis and mathematical morphology, Image Analysis and Mathematical Morphology Series, Academic Press, New York, 1982.

[10] E. Kasai, W. Rankin, R. Lovel, Y. Omori, An analysis of the structure of iron ore sinter cake, ISIJ International 29 (1989) 635-641.

[11] A. Molinari, J. Kazior, G. Straffelini, Investigation of liquid-phase sintering by image analysis, Materials characterization 34 (1995) 271-276.

[12] T. Marcu Puscas, M. Signorini, A. Molinari, G. Straffelini, Image analysis investigation of the effect of the process variables on the porosity of sintered chromium steels, Materials characterization 50 (2003) 1-10.

[13] J. Brzoska, B. Lesaffre, C. Coléou, K. Xu, R. Pieritz, Computation of 3D curvatures on a wet snow sample, The European Physical Journal Applied Physics 7 (1999) 45-57.

[14] F. Flin, J. Brzoska, B. Lesaffre, C. Coleou, R. Pieritz, Three-dimensional geometric measurements of snow microstructural evolution under isothermal conditions, Annals of glaciology 38 (2004) 39-44.

[15] F. Flin, J. Brzoska, B. Lesaffre, C. Coléou, R. Pieritz, Full three-dimensional modelling of curvature-dependent snow metamorphism: first results and comparison with experimental tomographic data, Journal of Physics D: Applied Physics 36 (2003) A49.

[16] H. de Oliveira Simões, A. M. Guilherme Bailon, F. de Jesus Alves, P. R. Santos Belarmino, W. Rangel Simões, T. Marchezi Doellinger, M. Cota Fonseca, Methodology development for determining the sintering degree in iron ore pellet grains through automatic image analysis, in: ICSTI, 6th International Congress on the Science and Technology of Ironmaking, 2012.

[17] Zeiss AxioVision, AxioVision for Materials: AxioVision - Perform to Perfection, Carl Zeiss Microscopy GmbH, Jena, Germany, 2009.

[18] MATLAB, version 8.0.0.783 (R2012b), The MathWorks Inc., Natick, Massachusetts, 2012.

[19] J. Schackelford, Introduction to material Science for Engineers, Prentice-Hall, Inc., New Jersey, USA, 1996.

[20] R. German, Sintering Theory and Practice (4th edition), John Wiley \& Sons Inc., New York, USA, 1996.

[21] E. Dougherty, R. Lotufo, Hands-on Morphological Image Processing, Spie press, Bellington, WA, USA, 2003. 
[22] R. Klette, A. Rosenfeld, Digital Geometry: Geometric Methods for Digital Picture Analysis, The Morgan Kaufmann Series in Computer Graphics, Morgan Kaufmann, Elsevier Science, San Francisco, 2004.

[23] I. Debled-Rennesson, J. Reveillès, A linear algorithm for segmentation of digital curves, International Journal of Pattern Recognition and Artificial Intelligence 9 (1995) 635-662.

[24] S. Hermann, R. Klette, A comparative study on 2D curvature estimators, in: Computing: Theory and Applications, 2007. ICCTA'07. International Conference on, IEEE, Kolkata, India, 2007, pp. 584-589.

[25] B. Kerautret, J.-O. Lachaud, B. Naegel, Curvature based corner detector for discrete, noisy and multi-scale contours, International Journal of Shape Modeling 14 (2008) 127-145.

[26] I. Debled-Rennesson, F. Feschet, J. Rouyer-Degli, Optimal blurred segments decomposition of noisy shapes in linear times, Computers and Graphics 30 (2006) 30-36.

[27] S. Forsmo, A. Apelqvist, B. Björkman, P.-O. Samskog, Binding mechanisms in wet iron ore green pellets with a bentonite binder, Powder technology 169 (2006) 147-158.

[28] F. S. Ray, Applied photographic optics (2nd edition), Focal press, Oxford, England, 1994.

[29] F. Nellros, M. Thurley, Automated image analysis of iron-ore pellet structure using optical microscopy, Minerals Engineering 24 (2011) 1525 - 1531.

[30] N. Otsu, A threshold selection method from gray-level histograms, IEEE Transactions on Systems, Man and Cybernetics 9 (1979) $62-66$.

[31] B. L. Welch, The generalization of "students" problem when several different population variances are involved, Biometrika 34 (1947) 28-35.

[32] V. Kovalevsky, Curvature in digital 2D images, International Journal of Pattern Recognition and Artificial Intelligence 15 (2001) 1183-1200. 